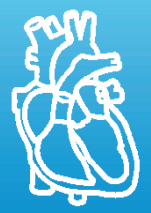

CARDIOLOGY
Mohammed V Military Teaching Hospital, Faculty of Medicine and Pharmacy Mohammed V University Rabat, Morocco
DOI: $10.15386 / \mathrm{mpr}-1254$

Manuscript received: 19.12.2018

Received in revised form: 22.05.2019

Accepted: 23.05.2019

Address for correspondence:

a.benelmekki@gmail.com

\section{Prosthetic thrombosis and coronary embolism during pregnancy: a delicate situation of antithrombotic therapy management}

Abdelilah Ben El Makki, Mohammed Malki, Hicham Faliouni, Mohammed Sabry

\begin{abstract}
Mechanical heart valve thrombosis is not so rare in pregnant women because of the difficulties in managing anticoagulant treatment and the hypercoagulability state associated with pregnancy. Among the embolic complications of valve prosthetic thrombosis, the coronary embolism is rare, it requires an urgent pharmaco-invasive approach which must be adapted to this particular associated condition. We report the observation and the difficulties of managing antithrombotic therapy in a pregnant patient hospitalized for non-obstructive prosthetic valve thrombosis complicated by coronary embolism and resulting in ST elevation myocardial infarction.
\end{abstract}

Keywords: prosthetic thrombosis; coronary embolism; pregnancy; antithrombotic therapy

\section{Introduction}

Pregnancy represents a state of hypercoagulability favoring the development of thrombosis, making the management of antithrombotic therapy problematic, especially in women with mechanical prosthetic valve. We report the observation of a pregnant woman with a mitral prosthetic valve thrombosis complicated by coronary embolism occurring after VKA substitution by LMWH.

\section{Observation}

A 28-year-old pregnant woman carrier of a mitral mechanical valve (Figure 1) had recently been put on enoxaparin 100 IU / kg twice daily in substitution of VKA, she was hospitalized for non obstructive prosthesis thrombus measuring $7 \mathrm{~mm}$ without prosthesis dysfunction (Figure 2 and 3), she was given unfractionated heparin at the dose of $500 \mathrm{ui} / \mathrm{kg} / 24 \mathrm{~h}$. On the third day of her hospitalization the patient complained of chest pain, the ECG showed ST segment elevation in inferior leads with mirror image in antero-septal and lateral leads (Figure 4). The patient was admitted to the cardiac catheterization room one hour later, coronarography showed a thrombotic occlusion of the marginal artery (Figure 5), which was removed by thromboaspiration, allowing the recovery of a TIMI2 flow. The total irradiation dose was $36 \mathrm{mGy}$.

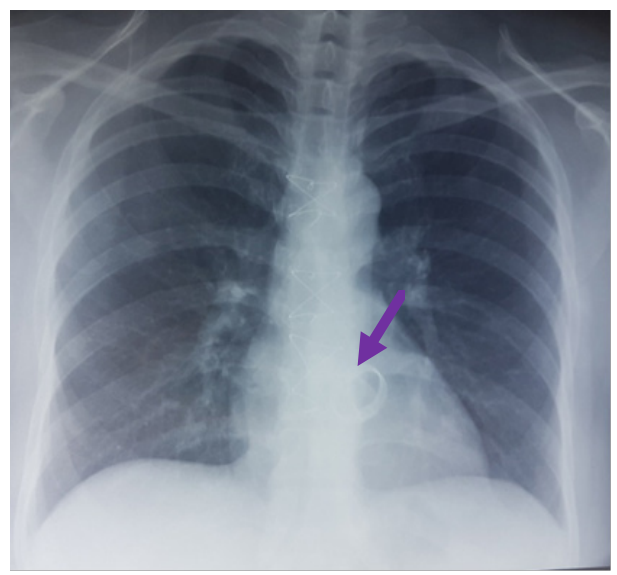

Figure 1. Chest X-ray with the mechanical valve.

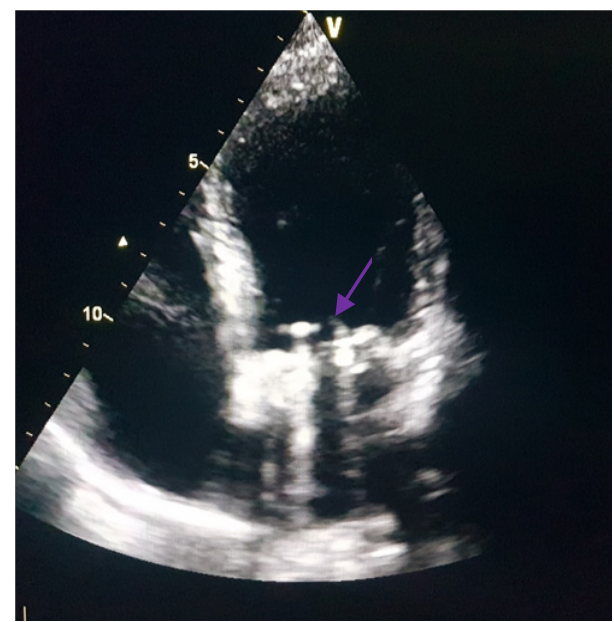

Figure 2. Transthoracic echocardiography: mitral prosthesis valve without signs of dysfunction. 


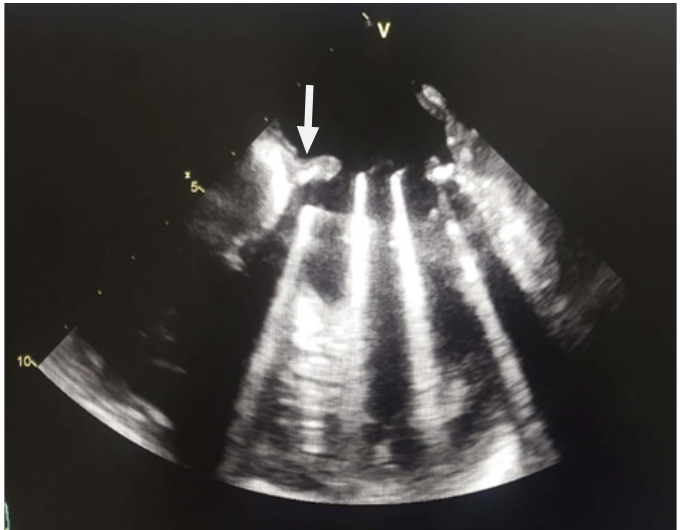

Figure 3. Transesophageal echocardiography showing thrombus image on mitral valve prosthesis (arrow).

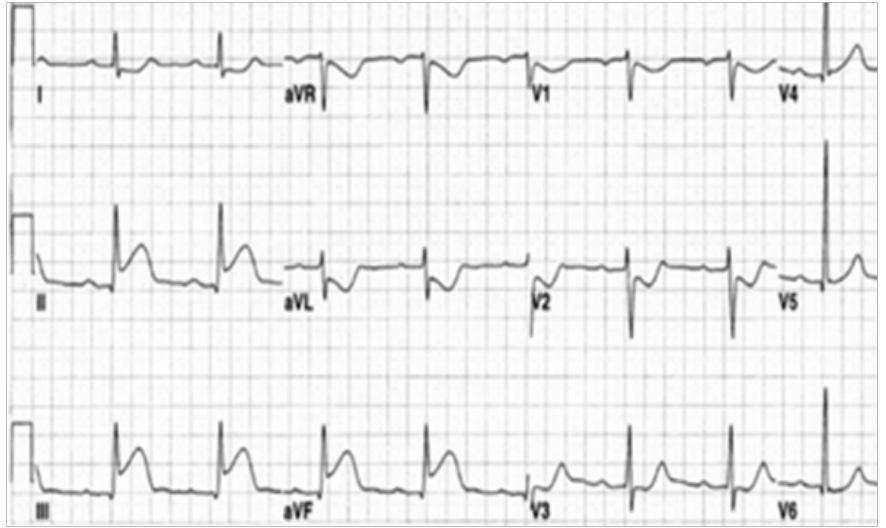

Figure 4. ECG showing ST elevation in inferior leads with mirror image in anterolateral leads.
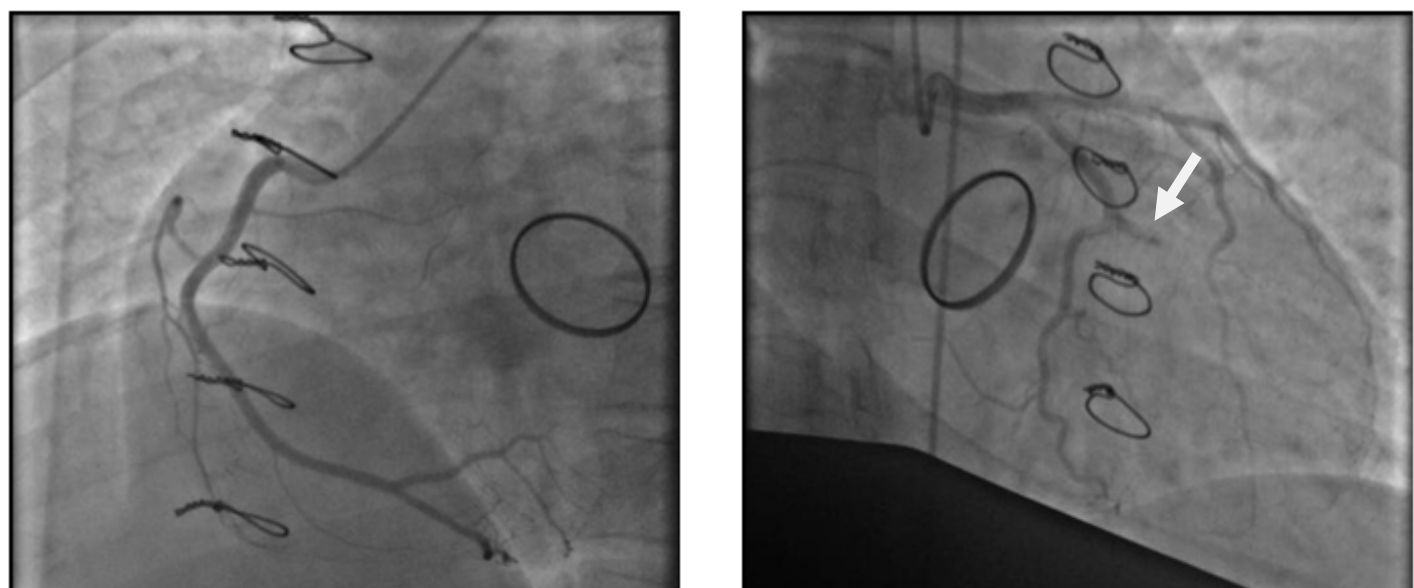

Figure 5. Coronary angiography showing a thrombotic occlusion of a marginal artery (arrow).

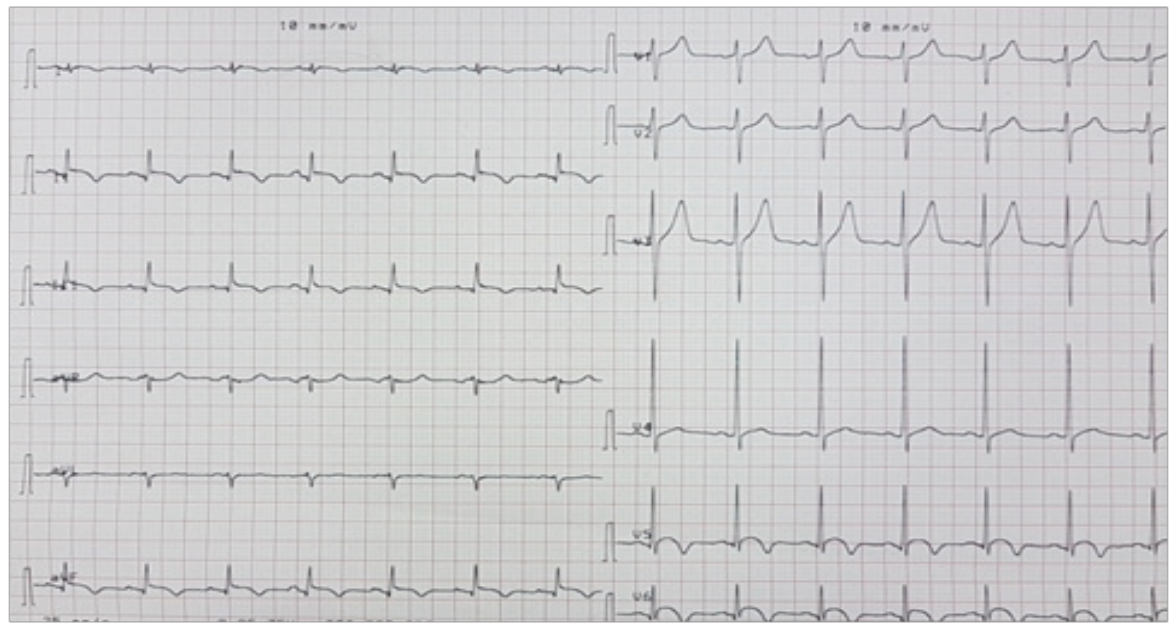

Figure 6. ECG after thromboaspiration showing ST segment regression with negative T waves.

ECG showed ST segment regression with $\mathrm{Q}$ waves in inferior leads (Figure 6).

Immediate transesophageal echocardiography visualized the persistence of a thrombus measured less than $5 \mathrm{~mm}$, the left ventricular function remained preserved with a $56 \%$ ejection fraction.

The patient was then given aspirin and unfractionated heparin. Glycoprotein IIb/IIIa inhibitors were not prescribed despite the fact that reperfusion was incomplete.

The control examination after one week showed 
complete thrombus dissolution.

After discussion with the patient to whom clear and precise information was given regarding the risks and benefit of each therapeutic option (VKA or Heparin), VKA was resumed with association of aspirin at the dose of $75 \mathrm{mg}$ daily.

The patient completed her pregnancy without incident and gave birth to a newborn male weighing $3 \mathrm{~kg}$ 200 , free from any malformation.

\section{Discussion}

Prosthetic valvethrombosisisacommoncomplication in pregnancy. It requires emergency management to avoid both fetal and maternal complications, the latter are often a cerebral embolism and prosthesis obstruction with acute pulmonary edema or even cardiogenic shock.

Coronary embolism, as in our patient's case, has rarely been reported in the literature [1], in such a situation fibrinolysis could be a treatment for both prosthetic thrombosis and its embolic complication; in our patient we opted rather for a coronarography with thromboaspiration which was immediately available to avoid a high hemorrhagic risk for the patient and her fetus, especially since the size of the thrombus was small.

Few studies have evaluated the safety of fibrinolysis in pregnant women. In a review of the literature including 172 pregnant women treated with fibrinolysis for various indications, the rate of bleeding complications reached $8.1 \%$ with maternal mortality of $1.2 \%$ and fetal mortality of $5.8 \%[2]$.

Glycoprotein IIb/IIIa inhibitors have not been sufficiently studied in pregnant women and their safety for the fetus is not clearly demonstrated therefore their use is not recommended [3].

Regarding the risk of irradiation for the fetus, there is no evidence for an increase in the rate of congenital malformations or pregnancy losses for an irradiation dose for the mother below 50 mGray $[4,5]$.

The remote management of these patients, with prosthetic thrombosis especially during the first trimester of pregnancy after replacement of VKA with heparin, often poses the problem of continuing the LMWH with a risk of new thrombosis, or resuming the VKA with a teratogenic risk for the fetus.

In a large review of the literature, the risk of prosthetic thrombosis during pregnancy was estimated at $3.9 \%$ with oral anticoagulants throughout the pregnancy, $9.2 \%$ when the UFH was used in the 1 st trimester relayed by the AVK during 2nd and 3rd trimester, and 33\% when UFH was used throughout pregnancy [6].

LMWHs are also associated with a higher risk of thrombosis as occurring in our patient, this risk can be decreased by monitoring anti-Xa activity allowing adjustment of doses which are often higher due to increased volume of distribution in pregnant women and increased renal clearance $[7,8]$.
In terms of fetal risk, VKA, unlike heparins, cross the placental barrier and may have teratogenic effects, particularly during the first trimester (0.6-1\%) [6]. This risk seems to be dose dependent as reported in a series where the risk is found to be of $2.6 \%$ when warfarin is used at a dose of less than $5 \mathrm{mg}$ against $8 \%$ when warfarin is used at a dose greater than $5 \mathrm{mg}$ [9].

\section{Conclusion}

Urgent management of acute coronary syndrome complicating prosthetic thrombosis in pregnancy is difficult. The choice of the anti-coagulation modality far from the acute episode of prosthetic thrombosis during the first trimester of pregnancy is challenging and must be discussed on a case-by-case basis, taking into account the patient's preferences after clear and precise information.

\section{References}

1. Varadarajan P, Isaeff D, Pai RG. Prosthetic valve thrombosis presenting as an acute embolic myocardial infarction in a pregnant patient: issues on anticoagulation regimens and thrombolytic therapy. Echocardiography. 2006;23:774-779.

2. Turrentine MA, Braems G, Ramirez MM. Use of thrombolytics for the treatment of thromboembolic disease during pregnancy. Obstet Gynecol Surv. 1995;50:534-541.

3. European Society of Gynecology (ESG); Association for European Paediatric Cardiology (AEPC); German Society for Gender Medicine (DGesGM), Regitz-Zagrosek V, Blomstrom Lundqvist C, Borghi C, Cifkova R, et al. ESC Guidelines on the management of cardiovascular diseases during pregnancy: the Task Force on the Management of Cardiovascular Diseases during Pregnancy of the European Society of Cardiology (ESC). Eur Heart J. 2011;32:3147-3197.

4. Brent RL. The effect of embryonic and fetal exposure to $\mathrm{x}$-ray, microwaves, and ultrasound: counseling the pregnant and nonpregnant patient about these risks. Semin Oncol. 1989; $16: 347-368$.

5. ACOG Committee on Obstetric Practice. ACOG Committee Opinion. Number 299, September 2004 (replaces No. 158, September 1995). Guidelines for diagnostic imaging during pregnancy. Obstet Gynecol. 2004;104:647-651.

6. Chan WS, Anand S, Ginsberg JS. Anticoagulation of pregnant women with mechanical heart valves: a systematic review of the literature. Arch Intern Med. 2000;160:191-196.

7. Quinn J, Von Klemperer K, Brooks R, Peebles D, Walker $\mathrm{F}$, Cohen H. Use of high intensity adjusted dose low molecular weight heparin in women with mechanical heart valves during pregnancy: a single-center experience. Haematologica. 2009;94:1608-1612.

8. Barbour LA, Oja JL, Schultz LK. A prospective trial that demonstrates that dalteparin requirements increase in pregnancy to maintain therapeutic levels of anticoagulation. Am J Obstet Gynecol. 2004;191:1024-1029.

9. Cotrufo M, De Feo M, De Santo LS, Romano G, Della Corte A, Renzulli A, et al. Risk of warfarin during pregnancy with mechanical valve prostheses. Obstet Gynecol. 2002;99:35-40. 